\title{
ALLELOPATHIC POTENTIAL OF LANTANA CAMARA AGAINST SELECTED WEEDS OF WHEAT CROP
}

\author{
ANWAR, T. ${ }^{1}$ - ILYAS, N. ${ }^{1}$ - QURESHI, R. $.^{*}-$ MAQSOOD, M. ${ }^{1}$ - MUNAZIR, M. ${ }^{2}$-ANWAR, ${ }^{3}{ }^{3}-$ \\ RAHIM, B. Z. ${ }^{4}-$ ANSARI, K. A. ${ }^{5}-$ PANNI, M. K. ${ }^{6}$ \\ ${ }^{1}$ Department of Botany, Pir Mehr Ali Shah Arid Agriculture University \\ Murree Road, Shamsabad, Rawalpindi 46300, Pakistan \\ ${ }^{2}$ Department of Botany, Government College Women University, Sialkot, Pakistan \\ ${ }^{3}$ Department of Biochemistry and Molecular Biology, University of Sialkot, Sialkot, Pakistan \\ ${ }^{4}$ Department of Botany, Baluchistan University, Quetta 87500, Baluchistan, Pakistan \\ ${ }^{5}$ Department of Botany, Shah Abdul Latif University, Khairpur, Sindh, Pakistan \\ ${ }^{6}$ Institiue of Biotechnology and Genetic Engineering (IBGE), The University of Agriculture \\ Peshawar 25130, Pakistan \\ *Corresponding author \\ e-mail: rahmatullahq@yahoo.com \\ (Received 6 Jul 2018; accepted 31 ${ }^{\text {st }}$ Aug 2018)
}

\begin{abstract}
The current study was aimed at screening allelopathic potential of Lantana camara L. against some noxious weeds (i.e. Phalaris minor, Avena fatua, Chenopodium album, Euphorbia helioscopia and Rumex dentatus) of wheat crop. The screening test of these weeds was carried out in laboratory on filter paper, soil and agar by three solvent extracts (viz., aqueous, hexane and methanolic) and sandwich method. The methanolic L. camara extract significantly suppressed germination percentage of $E$. helioscopia, $P$. minor $R$. dentatus and A. fatua measuring at 65\%,63\%, 61\% and 60\%, respectively. Likewise, L. camara significantly suppressed radical length of A. fatua, P. minor and C. album with 54\%, $53 \%$ and $52 \%$, respectively compared to the control. Besides, plumule length of E. helioscopia, C. album, $R$. dentatus and A. fatua was also inhibited at 56\%, 55\%, 53\% and 53\%, respectively by L. camara methanolic extract. It can be concluded that L. camara methanolic extract significantly reduced seed germination, radical and plumule length of selected weeds and have potential to be used in further detailed evaluation for searching bioherbicide from this plant.

Keywords: allelopathic activity, bio-herbicide, filter paper, noxious weed, sandwich method, screening test, wheat
\end{abstract}

\section{Introduction}

Wheat (Triticum aestivum L.) that belongs to family Poaceae, is one of the most important cereal crops of the world. It provides human nutrition to the most of human populations. Weeds are growing in cultivated crops and compete for light, moisture, other vital elements of nutrition and space and cause low quality and less productivity with raised costs of production (Arafat et al., 2015). Controlling weeds through traditional methods is time consuming with more labor cost. While weed control through synthetic chemicals, is expensive and hazardous that also cause environmental pollution (Dallali et al., 2017). This situation necessitates developing alternate weed management strategies (Thi et al., 2008). Certain plants produce many chemicals which are released by certain processes like, exudation, leaching and 
decomposition, etc. These chemicals can be obtained from different parts of certain plants like, flower, roots, stem and leaves. Such chemicals become a source of allelopathy, commonly called as allelochemicals. Allelopathy is a form of plant interference that can significantly influence ecosystem and agro ecosystem dynamics (Michelangelo et al., 2016). Allelochemicals come from the class of secondary metabolites. These are produced as byproducts in the primary metabolic ways of the plants (Anwar et al., 2013). Like many other natural compounds, these chemicals have the capacity of producing wide array of biological effects and can be quite useful for weed control processes as well as agriculture systems (Zhou et al., 2004).

Lantana camara L. belongs to Verbenaceae family. This plant possessed certain aromatic compounds which can inhibit early growth and seed germination of adjacent plant by outcompeting for soil nutrients and fluctuating micro environment by producing dense thickets (Dobhal et al., 2010). Keeping this in view, this study was undertaken for evaluating allelopathic activity of $L$. camara against some major weeds (viz., Phalaris minor, Avena fatua, Chenopodium album, Euphorbia helioscopia and Rumex dentatus) of wheat crop.

\section{Materials and methods}

\section{Collection and mechanical processing of L. camara}

This research was carried out at Plant Physiology Laboratory, Department of Botany, Pir Mehr Ali Shah Arid Agriculture University, Rawalpindi (PMAS-AAUR), Pakistan. The mature leaves of $L$. camara were collected from district Rawalpindi $\left(73^{\circ} 02^{\prime} \mathrm{E}\right.$ longitude and $33^{\circ} 36^{\prime} \mathrm{N}$ latitude, $508 \mathrm{~m}$ above sea level), Punjab, Pakistan and washed for several times under running tap water to remove dust. The same leaves were dried in blotting paper for 4 weeks in shade at room temperature (20$25^{\circ} \mathrm{C}$ ). The dried leaves were crushed using heavy duty blender (warring Lab.), to make fine powder, passed through mesh size $2 \mathrm{~mm}$ and kept in air tight plastic zip lock bags separately at $4{ }^{\circ} \mathrm{C}$ (Anwar et al., 2013).

\section{Procurement and surface sterilization of test species seeds}

Seeds of some noxious weeds sensitive to herbicide (Shahid et al., 2006) such as Avena fatua, Chenopodium album, Euphorbia helioscopia, Phalaris minor and Rumex dentatus and wheat (Wafaq-2001) were procured from the Barani Agricultural

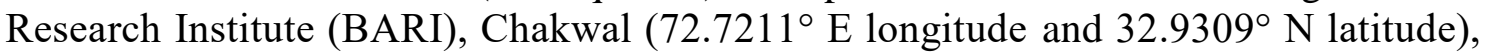
District Rawalpindi, Punjab, Pakistan. These weeds are reported to be the most densely populated weeds of wheat crop (Qureshi and Bhatti, 2001; Qureshi et al., 2009). Seeds were surface sterilized with $2 \%$ (w/v) solution of Sodium hypochlorite $(\mathrm{NaOCl})$ for 5-8 min. After disinfection, the seeds were washed several times with distilled water (Anwar et al., 2016).

\section{Studied parameters}

Subsequent three growth parameters were used for screening allelopathic potential as follows:
a. Germination percentage
b. Radical length $(\mathrm{cm})$
c. Plumule length $(\mathrm{cm})$ 


\section{Aqueous extract preparation}

An aliquot (10 g) of dried leaf powder of L. camara was soaked in $100 \mathrm{ml}$ distilled water in a flask and agitated at room temperature $\left(20-25^{\circ} \mathrm{C}\right)$ for $24 \mathrm{~h}$ on an orbital shaker $(160 \mathrm{rpm})$. The extract was strained through muslin cloth and finally filtered through Whatman filter paper No. 1. Aqueous extract was obtained as filtrate of the mixture and final volume was attuned to $100 \mathrm{ml}$, this gives 10\% water extract (Maharjan et al., 2007). This stock solution was then diluted with distilled water to prepare the different concentration of the extract i.e. $\mathrm{T}_{1}(100 \%), \mathrm{T}_{2}(75 \%), \mathrm{T}_{3}(50 \%)$ after Sahu and Devkota (2013). The control was distilled water $(0 \%)$, indicated as $\mathrm{T}_{4}$. The extract was stored at $4{ }^{\circ} \mathrm{C}$ in pre-disinfected flasks. To evade adulteration and forthcoming chemical modifications, the extracts were ensured to be used within 3-4 days (Anwar et al., 2017a).

\section{Hexane extract preparation}

An aliquot $(10 \mathrm{~g})$ of dried leaf powder of $L$. camara was mixed with hexane $(0.5 \mathrm{~L})$ repeated three times for $6 \mathrm{~h}$ on shaker. This extract was filtered, concentrated and dried over a rotary evaporator in pre-weighed flask. The obtained residue was $6.14 \mathrm{~g}$. The stock solution was then diluted with hexane to prepare three concentrations i.e. $\mathrm{T}_{1}(100 \%), \mathrm{T}_{2}$ (75\%), $\mathrm{T}_{3}(50 \%)$ and $\mathrm{T}_{4}\left(0 \%\right.$ control). These stored at $4{ }^{\circ} \mathrm{C}$ in pre-sterilized flasks (Sahu and Devkota, 2013).

\section{Methanolic extract preparation}

An aliquot (10 g) of dried leaf powder of $L$. camara placed on shaker with methanol (to extract polar compounds) $(0.5 \mathrm{~L})$ repeated three time for $6 \mathrm{~h}$ on shaker. This extract was filtered, concentrated, and dried over a rotary evaporator in pre-weighed flask. The obtained residue was $25.29 \mathrm{~g}$. The stock solution was than diluted with methanol to prepare three concentrations i.e. $\mathrm{T}_{1}(100 \%), \mathrm{T}_{2}(75 \%), \mathrm{T}_{3}(50 \%)$ and $\mathrm{T}_{4}(0 \%$ control). These stored at $4{ }^{\circ} \mathrm{C}$ in pre-sterilized flasks (Sahu and Devkota, 2013).

\section{Bioassay techniques}

An aliquot $(15 \mathrm{ml})$ of $L$. camara extract added on $25 \mathrm{~g}$ soil per Petri dish and $5 \mathrm{ml}$ extract was added on filter paper per Petri dish while distilled water, hexane and methanol used as control in aqueous, hexane and methanolic extraction respectively. Ten seeds of selected test species were used per Petri dish. Each treatment was replicated for five times. The Petri dishes were wrapped with squash tape, enclosed with Aluminum foil and incubated in the growth chamber (NTS Model MI-25S) at room temperature $\left(20-25^{\circ} \mathrm{C}\right)$ for 15 days. The germination percentages, lengths of radical and plumule were calculated of each test species by comparing with control (Khan et al., 2008).

\section{Sandwich method}

The sandwich method was followed after Fujii et al. (2003, 2004). Five ml of 0.75\% (w/v) agar (Nalge Nunc Intl., Roskilde, Denmark, gelling temperature $30-31^{\circ} \mathrm{C}$ ) was poured in each of the six-well $\left(10 \mathrm{~cm}^{2}\right.$ area /well) into multi-dish plastic plate. The agar solution was left for solidification. L. camara leaf powder @ 10 and $50 \mathrm{mg}$ were placed in wells of the plate and were roofed by a thin layer of $0.75 \%(w / v)$ agar. After solidification, 10 seeds of each test species were placed on agar gel in each well of the plate. The multi-well plastic plates were then wrapped with the plastic tape and incubated 
in the growth chamber (NTS Model MI-25S) at room temperature $\left(20-25^{\circ} \mathrm{C}\right)$ for 15 days. In the control treatment, only agar gel without dried leaves powder was used as a seed bed for test species seeds. Each treatment was replicated five times. The germination percentages, lengths of radical and plumule for each test species were calculated by comparing with control. An aliquot $(10 \mathrm{mg})$ of dried leaf powder of L. camara added on filter paper along with $5 \mathrm{ml}$ distilled water per Petri dish while an aliquot $(50 \mathrm{mg})$ of dried leaf powder of $L$. camara added on $25 \mathrm{~g}$ soil along with $15 \mathrm{ml}$ distilled water per Petri dish (Raana et al., 2012). Ten surface sterilized seeds of test species were placed to each sterilized Petri plate. The Petri dishes were wrapped with squash tape, enclosed with Aluminium foil and incubated in the growth chamber (NTS Model MI-25S) at room temperature $\left(20-25^{\circ} \mathrm{C}\right)$ for 15 days. The germination percentages, lengths of radical and plumule were calculated of each test species by comparing with control (Anwar et al., 2017b).

\section{Statistical analysis}

A completely randomized design (CRD) with five replications was used for the experiments. The statistical analysis was completed by STATISTIX 9 and means were separated by using Fisher's protected LSD test (Nekonam et al., 2014).

\section{Results}

\section{Allelopathic potential of L. camara aqueous extract}

\section{Germination percentage}

The results revealed that $L$. camara aqueous extract significantly inhibited germination percentage of $R$. dentatus (58\%), followed by E. helioscopia (56\%), A. fatua (57\%) and $P$. minor $(55 \%)$ on filter paper, whereas, no significant effect on germination percentage of T. aestivum and $C$. album (Table 1). The aqueous extract on soil significantly inhibited seed germination of E. helioscopia (64\%), followed by $P$. minor (63\%), $R$. dentatus (61\%) and A. fatua (60\%) as compared to control (Table 2). The results further indicated that maximum (96\% germination was observed for T. aestivum and C. album (Table 1). Besides, minimum germination was noted for $R$. dentatus $(42 \%)$ and E. helioscopia $(36 \%)$ on filter paper and soil, respectively (Tables 1 and 2). The statistics also recommended that germination percentage reduction of the $R$. dentatus, E. helioscopia, $A$. fatua and P. minor was concentration dependent (Fig. la).

Table 1. Allelopathic effect of $L$. camara aqueous extract $(A E)$ on germination percentage of test species on filter paper

\begin{tabular}{c|c|c|c|c|c|c}
\hline \multirow{2}{*}{ Treatments } & \multicolumn{6}{|c}{ Test species } \\
\cline { 2 - 7 } & T. aestivum & A. fatua & R. dentatus & P. minor & E. helioscopia & C. album \\
\hline $\mathrm{T}_{1 \mathrm{AE}}$ & $86^{\mathrm{a}}$ & $38^{\mathrm{d}}$ & $36^{\mathrm{d}}$ & $37^{\mathrm{d}}$ & $39^{\mathrm{d}}$ & $76^{\mathrm{a}}$ \\
$\mathrm{T}_{2 \mathrm{AE}}$ & $88^{\mathrm{a}}$ & $62^{\mathrm{c}}$ & $55 \mathrm{c}$ & $56^{\mathrm{c}}$ & $59^{\mathrm{c}}$ & $78^{\mathrm{a}}$ \\
$\mathrm{T}_{3 \mathrm{AE}}$ & $89^{\mathrm{a}}$ & $76^{\mathrm{b}}$ & $71^{\mathrm{b}}$ & $68^{\mathrm{b}}$ & $75^{\mathrm{b}}$ & $79^{\mathrm{a}}$ \\
$\mathrm{T}_{4 \mathrm{AE}}$ & $90^{\mathrm{a}}$ & $89^{\mathrm{a}}$ & $86^{\mathrm{a}}$ & $82^{\mathrm{a}}$ & $88^{\mathrm{a}}$ & $80^{\mathrm{a}}$ \\
$\mathrm{LSD}$ & 17.902 & 29.46 & 18.32 & 19.23 & 17.957 & 21.13 \\
$\mathrm{~F}-\mathrm{value}$ & $12.13^{*}$ & $18.29^{*}$ & $14.85^{*}$ & $31.44^{*}$ & $31.00^{*}$ & $17.61^{*}$ \\
\hline
\end{tabular}

Means followed by different letters within one column differ significantly at $\mathrm{P}<5 \%$

*Significant at $\mathrm{P}<1 \%$ 
Table 2. Allelopathic effect of L. camara aqueous extract (AE) on germination percentage of test species on soil

\begin{tabular}{c|c|c|c|c|c|c}
\hline \multirow{2}{*}{ Treatments } & \multicolumn{6}{|c}{ Test species } \\
\cline { 2 - 7 } & T. aestivum & A. fatua & R. dentatus & P. minor & E. helioscopia & C. album \\
\hline $\mathrm{T}_{1 \mathrm{AE}}$ & $87^{\mathrm{a}}$ & $36^{\mathrm{d}}$ & $34^{\mathrm{d}}$ & $31^{\mathrm{d}}$ & $32^{\mathrm{d}}$ & $77^{\mathrm{a}}$ \\
$\mathrm{T}_{2 \mathrm{AE}}$ & $89^{\mathrm{a}}$ & $52^{\mathrm{c}}$ & $52^{\mathrm{c}}$ & $49^{\mathrm{c}}$ & $51^{\mathrm{c}}$ & $79^{\mathrm{a}}$ \\
$\mathrm{T}_{3 \mathrm{AE}}$ & $90^{\mathrm{a}}$ & $71^{\mathrm{b}}$ & $69^{\mathrm{b}}$ & $60^{\mathrm{b}}$ & $69^{\mathrm{b}}$ & $80^{\mathrm{a}}$ \\
$\mathrm{T}_{4 \mathrm{AE}}$ & $91^{\mathrm{a}}$ & $90^{\mathrm{a}}$ & $87^{\mathrm{a}}$ & $83^{\mathrm{a}}$ & $89^{\mathrm{a}}$ & $81^{\mathrm{a}}$ \\
$\mathrm{LSD}$ & 18.608 & 14.197 & 19.778 & 17.604 & 14.541 & 18.12 \\
$\mathrm{~F}-\mathrm{value}$ & $10.89^{* *}$ & $42^{*}$ & $13.52^{*}$ & $14.67^{*}$ & $43.98^{*}$ & $14.65^{*}$ \\
\hline
\end{tabular}

Means followed by different letters within one column differ significantly at $P<5 \%$

*Significant at $P<1 \%$

\section{Radical length}

The data revealed that the highest radical length inhibition activity was exhibited by $A$. fatua (52\%), P. minor (51\%) and C. album (49\%) in L. camara aqueous extract on filter paper. The results further indicated that extract on soil caused significant radical length reduction of $A$. fatua (54\%), P. minor (53\%) and C. album (52\%) as compared to control. While the radical length of $T$. aestivum, $R$. dentatus and E. helioscopia remained unaffected. The results also illustrated that minimum radical length was noted for A. fatua i.e. $48 \%$ and $46 \%$ on filter paper and soil, respectively (Tables 3 and 4 ). The maximum radical length (97\%) was noted for T. aestivum, R. dentatus and E. helioscopia (Fig. 1b).

\section{Plumule length}

The data obtained exhibited that the aqueous extract of L. camara significantly inhibited the plumule length of E. helioscopia (52\%), A. fatua (50\%), R. dentatus (50\%) and $C$. album (50\%) as compared to control on filter paper. There was no significant effect on plumule elongation of T. aestivum and P. minor. Likewise, L. camara aqueous extract significantly inhibited plumule length of E. helioscopia (56\%), C. album (55\%), $R$. dentatus $(53 \%)$ and A. fatua (53\%) in soil. The statistical data concluded that minimum plumule length was noted for E. helioscopia i.e. $48 \%$ and $44 \%$ on filter paper and soil, respectively (Tables 5 and 6 ). The statistical figures also proposed that maximum plumule length (96\%) was noted for T. aestivum and P. minor (Fig. 1c).

Table 3. Allelopathic effect of $L$. camara aqueous extract (AE) on radical length ( $\mathrm{cm}$ ) of test species on filter paper

\begin{tabular}{c|c|c|c|c|c|c}
\hline \multirow{2}{*}{ Treatments } & \multicolumn{6}{|c}{ Test species } \\
\cline { 2 - 7 } & T. aestivum & A. fatua & R. dentatus & P. minor & E. helioscopia & C. album \\
\hline $\mathrm{T}_{1 \mathrm{AE}}$ & $9.01^{\mathrm{a}}$ & $4.46^{\mathrm{d}}$ & $8.11^{\mathrm{a}}$ & $4.62^{\mathrm{d}}$ & $7.15^{\mathrm{a}}$ & $4.10^{\mathrm{d}}$ \\
$\mathrm{T}_{2 \mathrm{AE}}$ & $9.05^{\mathrm{a}}$ & $6.71^{\mathrm{c}}$ & $8.16^{\mathrm{a}}$ & $6.45^{\mathrm{c}}$ & $7.19^{\mathrm{a}}$ & $5.52^{\mathrm{c}}$ \\
$\mathrm{T}_{3 \mathrm{AE}}$ & $9.05^{\mathrm{a}}$ & $8.02^{\mathrm{b}}$ & $8.19^{\mathrm{a}}$ & $7.92^{\mathrm{b}}$ & $7.22^{\mathrm{a}}$ & $6.78^{\mathrm{b}}$ \\
$\mathrm{T}_{4 \mathrm{AE}}$ & $9.11^{\mathrm{a}}$ & $9.25^{\mathrm{a}}$ & $8.21^{\mathrm{a}}$ & $9.42^{\mathrm{a}}$ & $7.28^{\mathrm{a}}$ & $8.11^{\mathrm{a}}$ \\
$\mathrm{LSD}$ & 1.7087 & 3.0879 & 1.0971 & 0.6573 & 1.0360 & 1.0027 \\
$\mathrm{~F}-\mathrm{value}$ & $47.38^{*}$ & $18.78^{*}$ & $126.95^{*}$ & $309.12^{*}$ & $128.48^{*}$ & $92.48^{*}$ \\
\hline
\end{tabular}

Means followed by different letters within one column differ significantly at $P<5 \%$

*Significant at $P<1 \%$ 
Table 4. Allelopathic effect of $L$. camara aqueous extract (AE) on radical length (cm) of test species on soil

\begin{tabular}{c|c|c|c|c|c|c}
\hline \multirow{2}{*}{ Treatments } & \multicolumn{6}{|c}{ Test species } \\
\cline { 2 - 7 } & T. aestivum & A. fatua & R. dentatus & P. minor & E. helioscopia & C. album \\
\hline $\mathrm{T}_{1 \mathrm{AE}}$ & $9.14^{\mathrm{a}}$ & $4.31^{\mathrm{d}}$ & $8.12^{\mathrm{a}}$ & $4.52^{\mathrm{d}}$ & $7.19^{\mathrm{a}}$ & $4.01^{\mathrm{c}}$ \\
$\mathrm{T}_{2 \mathrm{AE}}$ & $9.19^{\mathrm{a}}$ & $5.02^{\mathrm{c}}$ & $8.27^{\mathrm{a}}$ & $5.65^{\mathrm{b}}$ & $7.33^{\mathrm{a}}$ & $4.09^{\mathrm{c}}$ \\
$\mathrm{T}_{3 \mathrm{AE}}$ & $9.21^{\mathrm{a}}$ & $7.06^{\mathrm{b}}$ & $8.31^{\mathrm{a}}$ & $6.29^{\mathrm{b}}$ & $7.43^{\mathrm{a}}$ & $5.99^{\mathrm{b}}$ \\
$\mathrm{T}_{4 \mathrm{AE}}$ & $9.25^{\mathrm{a}}$ & $9.34^{\mathrm{a}}$ & $8.43^{\mathrm{a}}$ & $9.65^{\mathrm{a}}$ & $7.49^{\mathrm{a}}$ & $8.37^{\mathrm{a}}$ \\
$\mathrm{LSD}$ & 3.9879 & 1.8106 & 1.1918 & 1.0912 & 1.0634 & 0.8063 \\
$\mathrm{~F}-\mathrm{value}$ & $19.78^{*}$ & $52.46^{*}$ & $98.97^{*}$ & $83.01^{*}$ & $74.89^{*}$ & $126.70^{*}$ \\
\hline
\end{tabular}

Means followed by different letters within one column differ significantly at $P<5 \%$

*Significant at $P<1 \%$

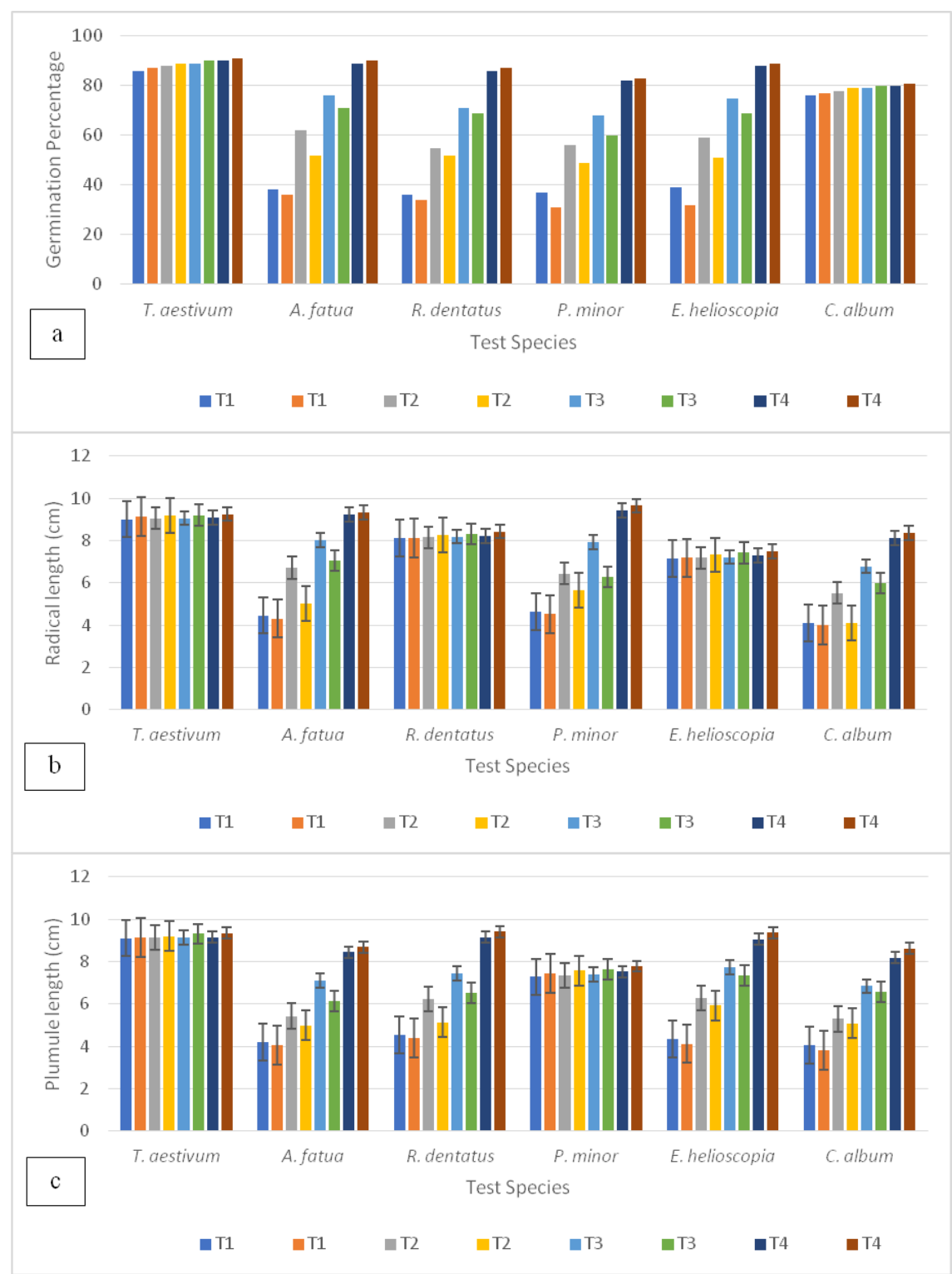

Figure 1. Allelopathic potential of L. camara aqueous extract against test species on filter paper (FP) and soil (S) on: (a) germination percentage, (b) radical length, (c) plumule length 
Table 5. Allelopathic effect of L. camara aqueous extract (AE) on plumule length (cm) of test species on filter paper

\begin{tabular}{c|c|c|c|c|c|c}
\hline \multirow{2}{*}{ Treatments } & \multicolumn{6}{|c}{ Test species } \\
\cline { 2 - 7 } & T. aestivum & A. fatua & R. dentatus & P. minor & E. helioscopia & C. album \\
\hline $\mathrm{T}_{1 \mathrm{AE}}$ & $9.11^{\mathrm{a}}$ & $4.20^{\mathrm{d}}$ & $4.53^{\mathrm{d}}$ & $7.28^{\mathrm{a}}$ & $4.33^{\mathrm{d}}$ & $4.06^{\mathrm{d}}$ \\
$\mathrm{T}_{2 \mathrm{AE}}$ & $9.13^{\mathrm{a}}$ & $5.43^{\mathrm{c}}$ & $6.24^{\mathrm{c}}$ & $7.33^{\mathrm{a}}$ & $6.28^{\mathrm{c}}$ & $5.29^{\mathrm{c}}$ \\
$\mathrm{T}_{3 \mathrm{AE}}$ & $9.15^{\mathrm{a}}$ & $7.09^{\mathrm{b}}$ & $7.46^{\mathrm{b}}$ & $7.41^{\mathrm{a}}$ & $7.73^{\mathrm{b}}$ & $6.84^{\mathrm{b}}$ \\
$\mathrm{T}_{4 \mathrm{AE}}$ & $9.16^{\mathrm{a}}$ & $8.45^{\mathrm{a}}$ & $9.15^{\mathrm{a}}$ & $7.53^{\mathrm{a}}$ & $9.06^{\mathrm{a}}$ & $8.19^{\mathrm{a}}$ \\
$\mathrm{LSD}$ & 1.3210 & 0.6647 & 1.0678 & 0.9468 & 0.6294 & 2.9953 \\
F-value & $45.63^{*}$ & $238.96^{*}$ & $97.24^{*}$ & $37.04^{*}$ & $197.36^{*}$ & $13.53^{*}$ \\
\hline
\end{tabular}

Means followed by different letters within one column differ significantly at $P<5 \%$

*Significant at $P<1 \%$

Table 6. Allelopathic effect of L. camara aqueous extract (AE) on plumule length (cm) of test species on soil

\begin{tabular}{c|c|c|c|c|c|c}
\hline \multirow{2}{*}{ Treatments } & \multicolumn{6}{|c}{ Test species } \\
\cline { 2 - 7 } & T. aestivum & A.fatua & R. dentatus & P. minor & E. helioscopia & C. album \\
\hline $\mathrm{T}_{1 \mathrm{AE}}$ & $9.13^{\mathrm{a}}$ & $4.06^{\mathrm{c}}$ & $4.38^{\mathrm{c}}$ & $7.43^{\mathrm{a}}$ & $4.12^{\mathrm{d}}$ & $3.81^{\mathrm{d}}$ \\
$\mathrm{T}_{2 \mathrm{AE}}$ & $9.21^{\mathrm{a}}$ & $4.98^{\mathrm{c}}$ & $5.12^{\mathrm{c}}$ & $7.58^{\mathrm{a}}$ & $5.92^{\mathrm{c}}$ & $5.09^{\mathrm{c}}$ \\
$\mathrm{T}_{3 \mathrm{AE}}$ & $9.32^{\mathrm{a}}$ & $6.14^{\mathrm{b}}$ & $6.52^{\mathrm{b}}$ & $7.64^{\mathrm{a}}$ & $7.34^{\mathrm{b}}$ & $6.58^{\mathrm{b}}$ \\
$\mathrm{T}_{4 \mathrm{AE}}$ & $9.34^{\mathrm{a}}$ & $8.68^{\mathrm{a}}$ & $9.41^{\mathrm{a}}$ & $7.78^{\mathrm{a}}$ & $9.37^{\mathrm{a}}$ & $8.62^{\mathrm{a}}$ \\
$\mathrm{LSD}$ & 2.9853 & 1.2127 & 0.6756 & 0.6851 & 0.8319 & 0.7647 \\
$\mathrm{~F}-\mathrm{value}$ & $12.53^{*}$ & $70.45^{*}$ & $117.35^{*}$ & $245.78^{*}$ & $209.87^{*}$ & $237.96^{*}$ \\
\hline
\end{tabular}

Means followed by different letters within one column differ significantly at $P<5 \%$

*Significant at $P<1 \%$

\section{Allelopathic potential of L. camara hexane extract}

\section{Germination percentage}

There was significant reduction of germination of E. helioscopia (35\%), followed by P. minor (33\%) and A. fatua (32\%) on filter paper (Table 7), whereas, no significant effect on the germination of $T$. aestivum, $R$. dentatus and $C$. album, showing resistance to the extract. The data illustrated that the highest seed germination inhibition was noted for P. minor (40\%), followed by A. fatua (39\%) and E. helioscopia (38\%) by the incorporation of $L$. camara hexane extract into soil. The maximum $(97 \%)$ germination noted for $T$. aestivum, $R$. dentatus and $C$. album. In the present study, minimum germination was noted for E. helioscopia $(65 \%)$ and P. minor $(60 \%)$ on filter paper and soil, respectively (Tables 7 and 8 ). The statistics also recommended that allelopathic inhibitory effect was concentration dependent for E. helioscopia, P. minor and A. fatua with concentration increase, suppression potential was gradually enhanced (Fig. 2a).

\section{Radical length}

The experimental results of the current study indicated that the highest radical length inhibition activity was exhibited by $A$. fatua $(38 \%)$ and $R$. dentatus (34\%) in L. camara hexane extract on filter paper. statistical data concluded that hexane extract on soil 
caused significant radical length reduction of A. fatua (40\%) and $R$. dentatus (39\%) as compared to control. The statistics also recommended that radical length of $T$. aestivum, P. minor, E. helioscopia and C. album remained unaffected. The results also illustrated that minimum radical length was noted for $A$. fatua i.e. $62 \%$ and $66 \%$ on filter paper and soil, respectively (Tables 9 and 10). The maximum radical length (96\%) was noted for T. T. aestivum, P. minor, E. helioscopia and C. album (Fig. 2b).

Table 7. Allelopathic effect of L. camara hexane extract (HE) on germination percentage of test species on filter paper

\begin{tabular}{c|c|c|c|c|c|c}
\hline \multirow{2}{*}{ Treatments } & \multicolumn{6}{|c}{ Test species } \\
\cline { 2 - 7 } & T. aestivum & A. fatua & R. dentatus & P. minor & E. helioscopia & C. album \\
\hline $\mathrm{T}_{1 \mathrm{HE}}$ & $86^{\mathrm{a}}$ & $63^{\mathrm{c}}$ & $82^{\mathrm{a}}$ & $55^{\mathrm{c}}$ & $60^{\mathrm{c}}$ & $76^{\mathrm{a}}$ \\
$\mathrm{T}_{2} \mathrm{HE}$ & $88^{\mathrm{a}}$ & $71^{\mathrm{c}}$ & $83^{\mathrm{a}}$ & $59^{\mathrm{c}}$ & $67^{\mathrm{c}}$ & $78^{\mathrm{a}}$ \\
$\mathrm{T}_{3} \mathrm{HE}$ & $89^{\mathrm{a}}$ & $82^{\mathrm{b}}$ & $84^{\mathrm{a}}$ & $71^{\mathrm{b}}$ & $80^{\mathrm{b}}$ & $79^{\mathrm{a}}$ \\
$\mathrm{T}_{4} \mathrm{HE}$ & $90^{\mathrm{a}}$ & $93^{\mathrm{a}}$ & $85^{\mathrm{a}}$ & $82^{\mathrm{a}}$ & $92^{\mathrm{a}}$ & $80^{\mathrm{a}}$ \\
$\mathrm{LSD}$ & 19.708 & 18.890 & 17.67 & 19.60 & 17.435 & 29.778 \\
F-value & $11.89^{* *}$ & $17.19^{*}$ & $32.71^{*}$ & $31.19^{*}$ & $21.97^{*}$ & $23.52^{*}$ \\
\hline
\end{tabular}

Means followed by different letters within one column differ significantly at $P<5 \%$

*Significant at $P<1 \%$

Table 8. Allelopathic effect of L. camara hexane extract (HE) on germination percentage of test species on soil

\begin{tabular}{c|c|c|c|c|c|c}
\hline \multirow{2}{*}{ Treatments } & \multicolumn{6}{|c}{ Test species } \\
\cline { 2 - 7 } & T. aestivum & A. fatua & R. dentatus & P. minor & E. helioscopia & C. album \\
\hline $\mathrm{T}_{1 \mathrm{HE}}$ & $89^{\mathrm{a}}$ & $58^{\mathrm{d}}$ & $84^{\mathrm{a}}$ & $50^{\mathrm{c}}$ & $58^{\mathrm{c}}$ & $78^{\mathrm{a}}$ \\
$\mathrm{T}_{2 \mathrm{HE}}$ & $90^{\mathrm{a}}$ & $68^{\mathrm{a}}$ & $85^{\mathrm{a}}$ & $58^{\mathrm{a}}$ & $64^{\mathrm{c}}$ & $80^{\mathrm{a}}$ \\
$\mathrm{T}_{3 \mathrm{HE}}$ & $91^{\mathrm{a}}$ & $80^{\mathrm{b}}$ & $86^{\mathrm{a}}$ & $68^{\mathrm{b}}$ & $74^{\mathrm{b}}$ & $81^{\mathrm{a}}$ \\
$\mathrm{T}_{4 \mathrm{HE}}$ & $92^{\mathrm{a}}$ & $95^{\mathrm{a}}$ & $87^{\mathrm{a}}$ & $84^{\mathrm{a}}$ & $94^{\mathrm{a}}$ & $82^{\mathrm{a}}$ \\
$\mathrm{LSD}$ & 13.554 & 17.510 & 16.580 & 14.744 & 14.08 & 19.13 \\
$\mathrm{~F}-$ value & $13.63^{*}$ & $22.36^{*}$ & $51.38^{*}$ & $21.04^{*}$ & $38.81^{*}$ & $31.14^{*}$ \\
\hline
\end{tabular}

Means followed by different letters within one column differ significantly at $\mathrm{P}<5 \%$

*Significant at $\mathrm{P}<1 \%$

Table 9. Allelopathic effect of $L$. camara hexane extract $(H E)$ on radical length $(\mathrm{cm})$ of test species on filter paper

\begin{tabular}{c|c|c|c|c|c|c}
\hline \multirow{2}{*}{ Treatments } & \multicolumn{6}{|c}{ Test species } \\
\cline { 2 - 7 } & T. aestivum & A. fatua & R. dentatus & P. minor & E. helioscopia & C. album \\
\hline $\mathrm{T}_{1 \mathrm{HE}}$ & $8.08^{\mathrm{a}}$ & $5.63^{\mathrm{d}}$ & $5.41^{\mathrm{c}}$ & $7.14^{\mathrm{a}}$ & $7.06^{\mathrm{a}}$ & $8.12^{\mathrm{a}}$ \\
$\mathrm{T}_{2 \mathrm{HE}}$ & $8.11^{\mathrm{a}}$ & $6.78^{\mathrm{c}}$ & $6.01^{\mathrm{c}}$ & $7.17^{\mathrm{a}}$ & $7.08^{\mathrm{a}}$ & $8.29^{\mathrm{a}}$ \\
$\mathrm{T}_{3 \mathrm{HE}}$ & $8.15^{\mathrm{a}}$ & $7.89^{\mathrm{b}}$ & $7.11^{\mathrm{b}}$ & $7.19^{\mathrm{a}}$ & $7.10^{\mathrm{a}}$ & $8.31^{\mathrm{a}}$ \\
$\mathrm{T}_{4 \mathrm{HE}}$ & $8.18^{\mathrm{a}}$ & $9.04^{\mathrm{a}}$ & $8.15^{\mathrm{a}}$ & $7.22^{\mathrm{a}}$ & $7.11^{\mathrm{a}}$ & $8.33^{\mathrm{a}}$ \\
$\mathrm{LSD}$ & 2.3332 & 1.4291 & 0.8679 & 1.2428 & 0.7319 & 0.8297 \\
$\mathrm{~F}-\mathrm{value}$ & $27.95^{*}$ & $23.83^{*}$ & $81.03^{*}$ & $29.85^{*}$ & $219.87^{*}$ & $222.41^{*}$ \\
\hline
\end{tabular}

Means followed by different letters within one column differ significantly at $\mathrm{P}<5 \%$

*Significant at $\mathrm{P}<1 \%$ 
Table 10. Allelopathic effect of L. camara hexane extract (HE) on radical length (cm) of test species on soil

\begin{tabular}{c|c|c|c|c|c|c}
\hline \multirow{2}{*}{ Treatments } & \multicolumn{6}{|c}{ Test species } \\
\cline { 2 - 7 } & T. aestivum & A. fatua & R. dentatus & P. minor & E. helioscopia & C. album \\
\hline $\mathrm{T}_{1 \mathrm{HE}}$ & $8.13^{\mathrm{a}}$ & $5.54^{\mathrm{c}}$ & $5.01^{\mathrm{c}}$ & $7.13^{\mathrm{a}}$ & $7.12^{\mathrm{a}}$ & $8.22^{\mathrm{a}}$ \\
$\mathrm{T}_{2} \mathrm{HE}$ & $8.19^{\mathrm{a}}$ & $6.48^{\mathrm{c}}$ & $5.89^{\mathrm{c}}$ & $7.26^{\mathrm{a}}$ & $7.14^{\mathrm{a}}$ & $8.39^{\mathrm{a}}$ \\
$\mathrm{T}_{3 \mathrm{HE}}$ & $8.23^{\mathrm{a}}$ & $7.61^{\mathrm{b}}$ & $6.99^{\mathrm{b}}$ & $7.31^{\mathrm{a}}$ & $7.17^{\mathrm{a}}$ & $8.41^{\mathrm{a}}$ \\
$\mathrm{T}_{4 \mathrm{HE}}$ & $8.28^{\mathrm{a}}$ & $9.24^{\mathrm{a}}$ & $8.25^{\mathrm{a}}$ & $7.32^{\mathrm{a}}$ & $7.21^{\mathrm{a}}$ & $8.43^{\mathrm{a}}$ \\
$\mathrm{LSD}$ & 2.4502 & 0.9978 & 0.7720 & 1.1434 & 0.8468 & 1.1019 \\
F-value & $9.54^{* *}$ & $72.85^{*}$ & $102.44^{*}$ & $72.9^{*}$ & $36.04^{*}$ & $86.41^{*}$ \\
\hline
\end{tabular}

Means followed by different letters within one column differ significantly at $\mathrm{P}<5 \%$

*Significant at $\mathrm{P}<1 \%$

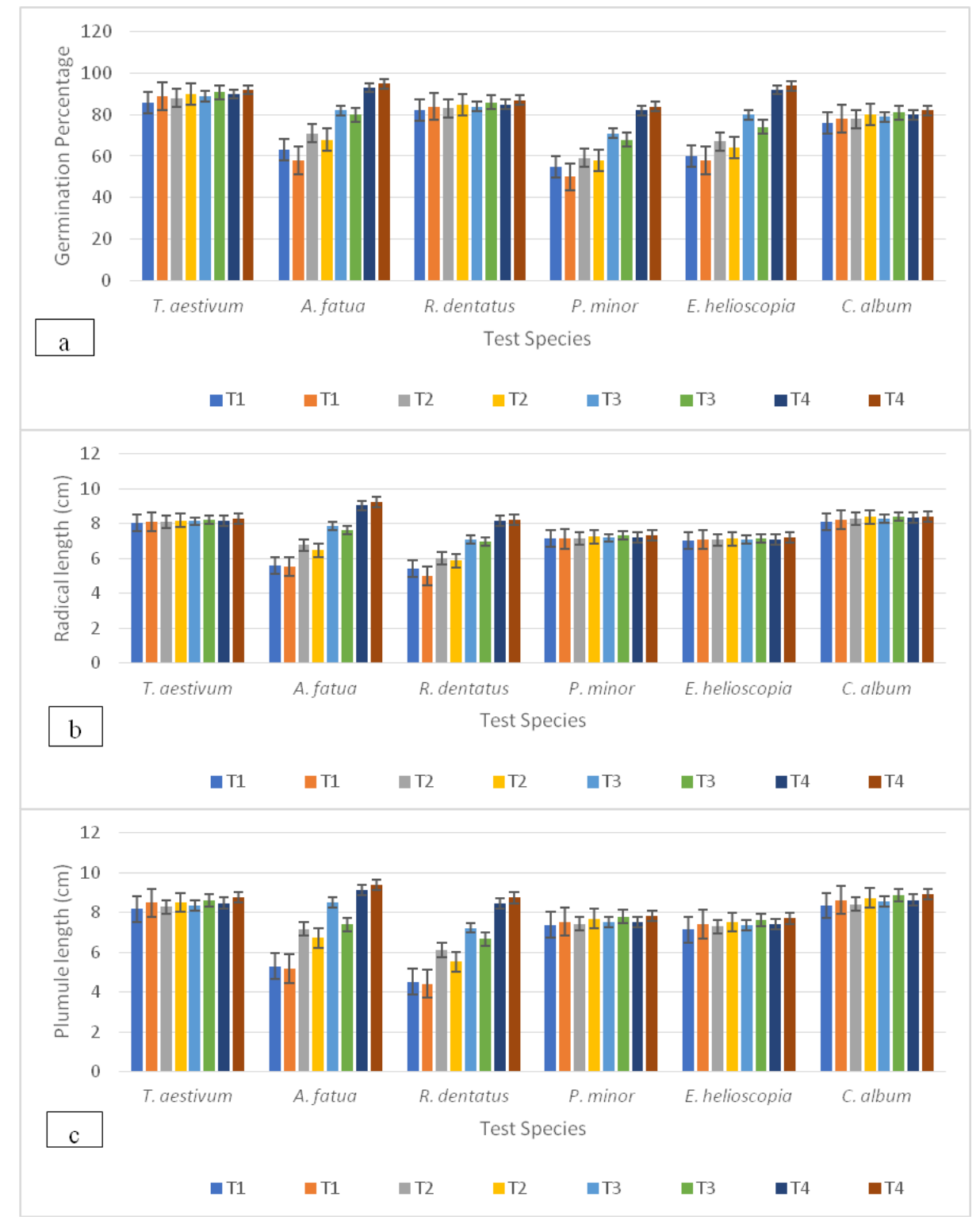

Figure 2. Allelopathic potential of L. camara hexane extract against test species on filter paper $(F P)$ and soil (S) on: (a) germination percentage (b) radical length (c) plumule length. 


\section{Plumule length}

Analysis of the data revealed that $L$. camara hexane extract significantly inhibited the plumule length of $R$. dentatus (46\%) and A. fatua (42\%) as compared to control on filter paper. Interestingly, there was no significant effect on plumule elongation of $T$. aestivum, P. minor, E. helioscopia and C. album. Likewise, L. camara hexane extract significantly inhibited plumule length of $R$. dentatus (49\%) and A. fatua (45\%) in soil. The statistical data concluded that minimum plumule length was noted for $R$. dentatus i.e., $54 \%$ and $51 \%$ on filter paper and soil, respectively (Tables 11 and 12). The statistical figures also proposed that maximum plumule length $(95 \%)$ was noted for $T$. aestivum, P. minor, E. helioscopia and C. album (Fig. 2c).

Table 11. Allelopathic effect of L. camara hexane extract (HE) on plumule length (cm) of test species on filter paper

\begin{tabular}{c|c|c|c|c|c|c}
\hline \multirow{2}{*}{ Treatments } & \multicolumn{6}{|c}{ Test species } \\
\cline { 2 - 7 } & T. aestivum & A. fatua & R. dentatus & P. minor & E. helioscopia & C. album \\
\hline $\mathrm{T}_{1 \mathrm{HE}}$ & $8.18^{\mathrm{a}}$ & $5.31^{\mathrm{d}}$ & $4.53^{\mathrm{d}}$ & $7.39^{\mathrm{a}}$ & $7.14^{\mathrm{a}}$ & $8.34^{\mathrm{a}}$ \\
$\mathrm{T}_{2 \mathrm{HE}}$ & $8.29^{\mathrm{a}}$ & $7.18^{\mathrm{c}}$ & $6.12^{\mathrm{c}}$ & $7.43^{\mathrm{a}}$ & $7.29^{\mathrm{a}}$ & $8.43^{\mathrm{a}}$ \\
$\mathrm{T}_{3 \mathrm{HE}}$ & $8.35^{\mathrm{a}}$ & $8.51^{\mathrm{b}}$ & $7.23^{\mathrm{b}}$ & $7.51^{\mathrm{a}}$ & $7.35^{\mathrm{a}}$ & $8.55^{\mathrm{a}}$ \\
$\mathrm{T}_{4 \mathrm{HE}}$ & $8.48^{\mathrm{a}}$ & $9.14^{\mathrm{a}}$ & $8.45^{\mathrm{a}}$ & $7.52^{\mathrm{a}}$ & $7.41^{\mathrm{a}}$ & $8.63^{\mathrm{a}}$ \\
$\mathrm{LSD}$ & 2.1332 & 1.5092 & 0.6679 & 1.5428 & 0.7497 & 0.6297 \\
$\mathrm{~F}-\mathrm{value}$ & $28.55^{*}$ & $24.81^{*}$ & $84.03^{*}$ & $27.95^{*}$ & $220.41^{*}$ & $221.91^{*}$ \\
\hline
\end{tabular}

Means followed by different letters within one column differ significantly at $\mathrm{P}<5 \%$

*Significant at $\mathrm{P}<1 \%$

Table 12. Allelopathic effect of L. camara hexane extract (HE) on plumule length (cm) of test species on soil

\begin{tabular}{c|c|c|c|c|c|c}
\hline \multirow{2}{*}{ Treatments } & \multicolumn{7}{c}{ Test species } \\
\cline { 2 - 7 } & T. aestivum & A. fatua & R. dentatus & P. minor & E. helioscopia & C. album \\
\hline $\mathrm{T}_{1 \mathrm{HE}}$ & $8.49^{\mathrm{a}}$ & $5.18^{\mathrm{c}}$ & $4.42^{\mathrm{d}}$ & $7.54^{\mathrm{a}}$ & $7.43^{\mathrm{a}}$ & $8.64^{\mathrm{a}}$ \\
$\mathrm{T}_{2 \mathrm{HE}}$ & $8.51^{\mathrm{a}}$ & $6.72^{\mathrm{b}}$ & $5.53^{\mathrm{c}}$ & $7.69^{\mathrm{a}}$ & $7.52^{\mathrm{a}}$ & $8.73^{\mathrm{a}}$ \\
$\mathrm{T}_{3 \mathrm{HE}}$ & $8.62^{\mathrm{a}}$ & $7.41^{\mathrm{b}}$ & $6.67^{\mathrm{b}}$ & $7.79^{\mathrm{a}}$ & $7.62^{\mathrm{a}}$ & $8.88^{\mathrm{a}}$ \\
$\mathrm{T}_{4 \mathrm{HE}}$ & $8.78^{\mathrm{a}}$ & $9.39^{\mathrm{a}}$ & $8.75^{\mathrm{a}}$ & $7.82^{\mathrm{a}}$ & $7.71^{\mathrm{a}}$ & $8.93^{\mathrm{a}}$ \\
$\mathrm{LSD}$ & 2.3501 & 0.8878 & 0.7630 & 1.6434 & 1.3019 & 0.5319 \\
F-value & $9.65^{* *}$ & $75.85^{*}$ & $101.54^{*}$ & $73.1^{*}$ & $84.41^{*}$ & $239.87^{*}$ \\
\hline
\end{tabular}

Means followed by different letters within one column differ significantly at $\mathrm{P}<5 \%$

*Significant at $\mathrm{P}<1 \%$

\section{Allelopathic potential of L. camara methanolic extract}

\section{Germination percentage}

The statistical data exposed that $L$. camara methanolic extract significantly inhibited germination of $R$. dentatus (68\%), A. fatua (66\%), E. helioscopia (63\%) and P. minor (62\%) on filter paper, whereas, no significant effect on germination of $T$. aestivum and C. album showing resistance against extract. Similarly, L. camara methanolic extract on soil significantly suppressed seed germination of A. fatua (76\%), E. helioscopia (73\%), 
$R$. dentatus $(70 \%)$ and $P$. minor $(69 \%)$. It was noted that maximum $(95 \%)$ germination was observed for T. aestivum and C. album. In the present study, it was recognized that minimum germination was noted for $R$. dentatus (32\%) followed by A. fatua (24\%) on filter paper and soil, respectively (Tables 13 and 14). The results revealed that germination reduction of the $R$. dentatus, A. fatua, E. helioscopia and $P$. minor were concentration dependent (Fig. 3a).

Table 13. Allelopathic effect of $L$. camara methanolic extract $(M E)$ on germination percentage of test species on filter paper

\begin{tabular}{c|c|c|c|c|c|c}
\hline \multirow{2}{*}{ Treatments } & \multicolumn{6}{|c}{ Test species } \\
\cline { 2 - 7 } & T. aestivum & A. fatua & R. dentatus & P. minor & E. helioscopia & C. album \\
\hline $\mathrm{T}_{1 \mathrm{ME}}$ & $88^{\mathrm{a}}$ & $31^{\mathrm{d}}$ & $28^{\mathrm{d}}$ & $32^{\mathrm{d}}$ & $33^{\mathrm{d}}$ & $78^{\mathrm{a}}$ \\
$\mathrm{T}_{2 \mathrm{ME}}$ & $90^{\mathrm{a}}$ & $56^{\mathrm{c}}$ & $58^{\mathrm{c}}$ & $54^{\mathrm{c}}$ & $51^{\mathrm{c}}$ & $80^{\mathrm{a}}$ \\
$\mathrm{T}_{3 \mathrm{ME}}$ & $91^{\mathrm{a}}$ & $75^{\mathrm{b}}$ & $74^{\mathrm{b}}$ & $69^{\mathrm{b}}$ & $70^{\mathrm{b}}$ & $81^{\mathrm{a}}$ \\
$\mathrm{T}_{4 \mathrm{ME}}$ & $92^{\mathrm{a}}$ & $91^{\mathrm{a}}$ & $88^{\mathrm{a}}$ & $84^{\mathrm{a}}$ & $90^{\mathrm{a}}$ & $82^{\mathrm{a}}$ \\
$\mathrm{LSD}$ & 18.65 & 18.702 & 15.197 & 39.778 & 18.604 & 16.541 \\
$\mathrm{~F}-\mathrm{value}$ & $15.19^{*}$ & $13.13^{*}$ & $42.98^{*}$ & $33.52^{*}$ & $16.67^{*}$ & $45.98^{*}$ \\
\hline
\end{tabular}

Means followed by different letters within one column differ significantly at $\mathrm{P}<5 \%$

*Significant at $\mathrm{P}<1 \%$

Table 14. Allelopathic effect of L. camara methanolic extract $(M E)$ on germination percentage of test species on soil

\begin{tabular}{c|c|c|c|c|c|c}
\hline \multirow{2}{*}{ Treatments } & \multicolumn{6}{|c}{ Test species } \\
\cline { 2 - 7 } & T. aestivum & A. fatua & R. dentatus & P. minor & E. helioscopia & C. album \\
\hline $\mathrm{T}_{1 \mathrm{ME}}$ & $90^{\mathrm{a}}$ & $22^{\mathrm{d}}$ & $27^{\mathrm{d}}$ & $26^{\mathrm{d}}$ & $25^{\mathrm{d}}$ & $79^{\mathrm{a}}$ \\
$\mathrm{T}_{2 \mathrm{ME}}$ & $91^{\mathrm{a}}$ & $49^{\mathrm{c}}$ & $48^{\mathrm{c}}$ & $42^{\mathrm{c}}$ & $47^{\mathrm{c}}$ & $81^{\mathrm{a}}$ \\
$\mathrm{T}_{3 \mathrm{ME}}$ & $92^{\mathrm{a}}$ & $69^{\mathrm{b}}$ & $68^{\mathrm{b}}$ & $61^{\mathrm{b}}$ & $62^{\mathrm{b}}$ & $82^{\mathrm{a}}$ \\
$\mathrm{T}_{4 \mathrm{ME}}$ & $93^{\mathrm{a}}$ & $92^{\mathrm{a}}$ & $89^{\mathrm{a}}$ & $85^{\mathrm{a}}$ & $91^{\mathrm{a}}$ & $83^{\mathrm{a}}$ \\
$\mathrm{LSD}$ & 11.554 & 17.857 & 15.18 & 14.944 & 16.480 & 15.61 \\
F-value & $13.83^{*}$ & $31.80^{*}$ & $38.99^{*}$ & $21.14^{*}$ & $51.28^{*}$ & $25.18^{*}$ \\
\hline
\end{tabular}

Means followed by different letters within one column differ significantly at $\mathrm{P}<5 \%$

*Significant at $\mathrm{P}<1 \%$

\section{Radical length}

The results obtained in the study indicated that $L$. camara methanolic extract significantly inhibited radical length of C. album (60\%), P. minor (63\%) and A. fatua $(66 \%)$ on filter paper, whereas, no significant effect was noted for $T$. aestivum, $R$. dentatus and E. helioscopia showing resistance against extract. Similarly, the applications of extract into soil significantly suppressed radical length of A. fatua (68\%), P. minor (66\%) C. album (63\%) and E. helioscopia (55\%) as compared to control (Tables 15 and 16). The maximum (96\%) radical length was observed for $T$. aestivum and $R$. dentatus. The final data concluded that minimum radical length was noted for C. album (40\%) and A. fatua (32\%) on filter paper and soil, respectively (Fig. 3b). 


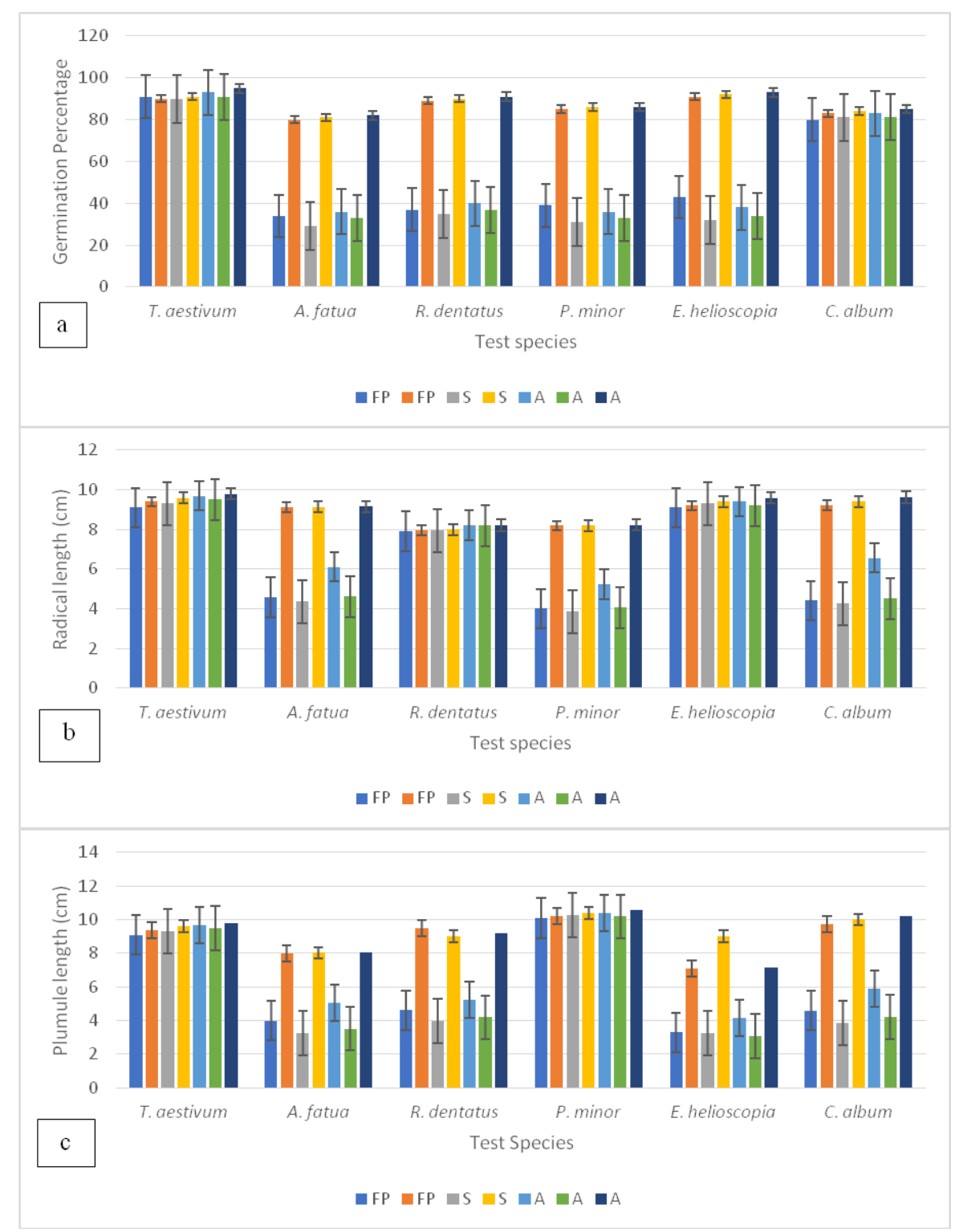

Figure 3. Allelopathic potential of L. camara leaf powder against test species on filter paper $(F P)$, soil (S) and agar (A) on: (a) germination percentage (b) radical length (c) plumule length

Table 15. Allelopathic effect of L. camara methanolic extract (ME) on radical length ( $\mathrm{cm}$ ) of test species on filter paper

\begin{tabular}{c|c|c|c|c|c|c}
\hline \multirow{2}{*}{ Treatments } & \multicolumn{6}{|c}{ Test species } \\
\cline { 2 - 7 } & T. aestivum & A. fatua & R. dentatus & P. minor & E. helioscopia & C. album \\
\hline $\mathrm{T}_{1 \mathrm{ME}}$ & $9.11^{\mathrm{a}}$ & $3.18^{\mathrm{d}}$ & $8.31^{\mathrm{a}}$ & $3.59^{\mathrm{d}}$ & $7.49^{\mathrm{a}}$ & $3.39^{\mathrm{d}}$ \\
$\mathrm{T}_{2 \mathrm{ME}}$ & $9.23^{\mathrm{a}}$ & $6.24^{\mathrm{c}}$ & $8.43^{\mathrm{a}}$ & $6.88^{\mathrm{c}}$ & $7.51^{\mathrm{a}}$ & $5.46^{\mathrm{c}}$ \\
$\mathrm{T}_{3 \mathrm{ME}}$ & $9.37^{\mathrm{a}}$ & $7.93^{\mathrm{b}}$ & $8.54^{\mathrm{a}}$ & $8.10^{\mathrm{b}}$ & $7.63^{\mathrm{a}}$ & $7.11^{\mathrm{b}}$ \\
$\mathrm{T}_{4 \mathrm{ME}}$ & $9.43^{\mathrm{a}}$ & $9.49^{\mathrm{a}}$ & $8.67^{\mathrm{a}}$ & $9.81^{\mathrm{a}}$ & $7.69^{\mathrm{a}}$ & $8.58^{\mathrm{a}}$ \\
$\mathrm{LSD}$ & 3.7879 & 2.5542 & 0.8095 & 1.2569 & 1.9073 & 0.9043 \\
$\mathrm{~F}-\mathrm{value}$ & $16.78^{*}$ & $23.58^{*}$ & $206.09^{*}$ & $39.32^{*}$ & $27.90^{*}$ & $20.88^{*}$ \\
\hline
\end{tabular}

Means followed by different letters within one column differ significantly at $\mathrm{P}<5 \%$

*Significant at $\mathrm{P}<1 \%$ 
Table 16. Allelopathic effect of $L$. camara methanolic extract $(M E)$ on radical length $(\mathrm{cm})$ of test species on soil

\begin{tabular}{c|c|c|c|c|c|c}
\hline \multirow{2}{*}{ Treatments } & \multicolumn{6}{|c}{ Test species } \\
\cline { 2 - 7 } & T. aestivum & A. fatua & R. dentatus & P. minor & E. helioscopia & C. album \\
\hline $\mathrm{T}_{1 \mathrm{ME}}$ & $9.46^{\mathrm{a}}$ & $3.07^{\mathrm{c}}$ & $8.52^{\mathrm{a}}$ & $3.35^{\mathrm{d}}$ & $3.51^{\mathrm{a}}$ & $3.31^{\mathrm{d}}$ \\
$\mathrm{T}_{2 \mathrm{ME}}$ & $9.51^{\mathrm{a}}$ & $5.45^{\mathrm{b}}$ & $8.66^{\mathrm{a}}$ & $5.28^{\mathrm{c}}$ & $7.65^{\mathrm{a}}$ & $4.49^{\mathrm{c}}$ \\
$\mathrm{T}_{3 \mathrm{ME}}$ & $9.61^{\mathrm{a}}$ & $6.03^{\mathrm{b}}$ & $8.77^{\mathrm{a}}$ & $7.31^{\mathrm{b}}$ & $7.72^{\mathrm{a}}$ & $6.45^{\mathrm{b}}$ \\
$\mathrm{T}_{4 \mathrm{ME}}$ & $9.65^{\mathrm{a}}$ & $9.56^{\mathrm{a}}$ & $8.89^{\mathrm{a}}$ & $9.99^{\mathrm{a}}$ & $7.87^{\mathrm{a}}$ & $8.85^{\mathrm{a}}$ \\
$\mathrm{LSD}$ & 2.4770 & 1.740 & 0.989 & 1.3125 & 1.2662 & 2.420 \\
F-value & $18.01^{*}$ & $44.47^{*}$ & $64.33^{*}$ & $121.84^{*}$ & $94.32^{*}$ & $53.72^{*}$ \\
\hline
\end{tabular}

Means followed by different letters within one column differ significantly at $\mathrm{P}<5 \%$

*Significant at $\mathrm{P}<1 \%$

\section{Plumule length}

The data obtained exhibited that L. camara methanolic extract significantly suppressed the plumule length of $R$. dentatus (63\%), A. fatua (63\%), C. album (61\%) and E. helioscopia (59\%) as compared control on filter paper. Interestingly, there was no significant effect on plumule elongation of $T$. aestivum and $P$. minor. Likewise, $L$. camara methanolic extract significantly inhibited plumule length of E. helioscopia (67\%), R. dentatus (66\%), A. fatua (65\%) and C. album (64\%) in soil. The statistical data concluded that minimum plumule length was noted for $R$. dentatus $(37 \%)$ and $E$. helioscopia (33\%) on filter paper and soil, respectively (Tables 17 and 18). The statistical figures also proposed that maximum plumule length (96\%) was noted for $T$. aestivum and P. minor (Fig. 3c).

\section{Allelopathic potential of L. camara leaf powder}

\section{Germination percentage}

The data revealed that A. fatua (60\%), R. dentatus (58\%), P. minor (54\%) and E. helioscopia (53\%) possessed significant germination in L. camara leaf powder on filter paper, whereas, no significant effect on germination of $T$. aestivum and C. album showing resistance to dry powder. It is also clear from the result that E. helioscopia (65\%), P. minor (64\%), A. fatua (64\%) and $R$. dentatus (61\%) showed inhibition respectively as compared to control in powder applied into soil. The results also declared that maximum (95\%) germination was noted for T. aestivum and C. album. In the present study, it was demonstrated that minimum germination was noted for $A$. fatua (42\%) and E. helioscopia (45\%) on filter paper and soil, respectively. The experimental results of the current study indicated on agar the highest germination reduction was noted for E. helioscopia (59\%), followed by P. minor (58\%), R. dentatus (56\%) and A. fatua $(56 \%)$ at $10 \mathrm{mg}$ conc. Similarly, the highest germination reduction was noted for E. helioscopia (63\%), followed by P. minor (62\%), A. fatua (60\%) and $R$. dentatus $(59 \%)$ at $50 \mathrm{mg}$ conc. The statistical data concluded that minimum germination was noted for E. helioscopia i.e. $41 \%$ and $37 \%$ at $10 \mathrm{mg}$ and at $50 \mathrm{mg}$ conc., respectively. The statistics also recommended that with the increase of concentration, the inhibitory effect was progressively increased for E. helioscopia, P. minor, A. fatua and R. dentatus 
(Table 19). The statistical results recommended that the germination \%age T. aestivum and C. album were completely resistant to dry powder (Fig. 4a).

Table 17. Allelopathic effect of L. camara methanolic extract $(M E)$ on plumule length $(\mathrm{cm})$ of test species on filter paper

\begin{tabular}{c|c|c|c|c|c|c}
\hline \multirow{2}{*}{ Treatments } & \multicolumn{7}{c}{ Test species } \\
\cline { 2 - 7 } & T. aestivum & A. fatua & R. dentatus & P. minor & E. helioscopia & C. album \\
\hline $\mathrm{T}_{1 \mathrm{ME}}$ & $9.33^{\mathrm{a}}$ & $3.19^{\mathrm{d}}$ & $3.42^{\mathrm{d}}$ & $7.31^{\mathrm{a}}$ & $3.71^{\mathrm{d}}$ & $3.26^{\mathrm{d}}$ \\
$\mathrm{T}_{2 \mathrm{ME}}$ & $9.41^{\mathrm{a}}$ & $5.97^{\mathrm{c}}$ & $6.38^{\mathrm{c}}$ & $7.44^{\mathrm{a}}$ & $6.28^{\mathrm{c}}$ & $5.64^{\mathrm{c}}$ \\
$\mathrm{T}_{3 \mathrm{ME}}$ & $9.50^{\mathrm{a}}$ & $7.33^{\mathrm{b}}$ & $7.92^{\mathrm{b}}$ & $7.59^{\mathrm{a}}$ & $7.91^{\mathrm{b}}$ & $7.18^{\mathrm{b}}$ \\
$\mathrm{T}_{4 \mathrm{ME}}$ & $9.59^{\mathrm{a}}$ & $8.55^{\mathrm{a}}$ & $9.27^{\mathrm{a}}$ & $7.61^{\mathrm{a}}$ & $9.16^{\mathrm{a}}$ & $8.29^{\mathrm{a}}$ \\
$\mathrm{LSD}$ & 1.4210 & $0.6947^{\mathrm{a}}$ & 1.4678 & 0.6468 & 0.9294 & 1.6091 \\
F-value & $43.63^{*}$ & $228.96^{*}$ & $95.24^{*}$ & $35.04^{*}$ & $193.36^{*}$ & $25.73^{*}$ \\
\hline
\end{tabular}

Means followed by different letters within one column differ significantly at $\mathrm{P}<5 \%$

*Significant at $\mathrm{P}<1 \%$

Table 18. Allelopathic effect of L. camara methanolic extract $(M E)$ on plumule length $(\mathrm{cm})$ of test species on soil

\begin{tabular}{c|c|c|c|c|c|c}
\hline \multirow{2}{*}{ Treatments } & \multicolumn{6}{|c}{ Test species } \\
\cline { 2 - 7 } & T. aestivum & A. fatua & R. dentatus & P. minor & E. helioscopia & C. album \\
\hline $\mathrm{T}_{1 \mathrm{ME}}$ & $9.41^{\mathrm{a}}$ & $3.09^{\mathrm{d}}$ & $3.27^{\mathrm{d}}$ & $7.56^{\mathrm{a}}$ & $3.11^{\mathrm{d}}$ & $3.11^{\mathrm{d}}$ \\
$\mathrm{T}_{2 \mathrm{ME}}$ & $9.53^{\mathrm{a}}$ & $4.55^{\mathrm{c}}$ & $5.79^{\mathrm{c}}$ & $7.68^{\mathrm{a}}$ & $5.12^{\mathrm{c}}$ & $5.23^{\mathrm{c}}$ \\
$\mathrm{T}_{3 \mathrm{ME}}$ & $9.65^{\mathrm{a}}$ & $7.08^{\mathrm{b}}$ & $7.12^{\mathrm{b}}$ & $7.79^{\mathrm{a}}$ & $7.34^{\mathrm{b}}$ & $7.09^{\mathrm{b}}$ \\
$\mathrm{T}_{4 \mathrm{ME}}$ & $9.75^{\mathrm{a}}$ & $8.87^{\mathrm{a}}$ & $9.59^{\mathrm{a}}$ & $7.81^{\mathrm{a}}$ & $9.47^{\mathrm{a}}$ & $8.73^{\mathrm{a}}$ \\
$\mathrm{LSD}$ & 2.8953 & 1.3127 & 0.6726 & 0.6941 & 0.6419 & 0.5756 \\
$\mathrm{~F}-\mathrm{value}$ & $15.53^{*}$ & $71.45^{*}$ & $117.15^{*}$ & $235.78^{*}$ & $225.87^{*}$ & $119.35^{*}$ \\
\hline
\end{tabular}

Means followed by different letters within one column differ significantly at $\mathrm{P}<5 \%$

*Significant at $\mathrm{P}<1 \%$

Table 19. Allelopathic effect of L. camara leaf powder on germination percentage of test species

\begin{tabular}{c|c|c|c|c|c|c|c}
\hline \multicolumn{2}{c|}{ Treatments } & \multicolumn{6}{c}{ Test species } \\
\cline { 3 - 8 } \multicolumn{2}{c|}{ T. aestivum } & A. fatua & R. dentatus & P. minor & E. helioscopia & \multirow{2}{*}{ C. album } \\
\hline Media & & & & & & & \\
\hline Filter & $10 \mathrm{mg}$ & $91^{\mathrm{a}}$ & $34^{\mathrm{b}}$ & $37^{\mathrm{b}}$ & $39^{\mathrm{b}}$ & $43^{\mathrm{b}}$ & $80^{\mathrm{a}}$ \\
paper & Control & $90^{\mathrm{a}}$ & $80^{\mathrm{a}}$ & $89^{\mathrm{a}}$ & $85^{\mathrm{a}}$ & $91^{\mathrm{a}}$ & $83^{\mathrm{a}}$ \\
\hline \multirow{2}{*}{ Soil } & $50 \mathrm{mg}$ & $90^{\mathrm{a}}$ & $29^{\mathrm{b}}$ & $35^{\mathrm{b}}$ & $31^{\mathrm{b}}$ & $32^{\mathrm{b}}$ & $81^{\mathrm{a}}$ \\
& Control & $91^{\mathrm{a}}$ & $81^{\mathrm{a}}$ & $90^{\mathrm{a}}$ & $86^{\mathrm{a}}$ & $92^{\mathrm{a}}$ & $84^{\mathrm{a}}$ \\
\hline & $10 \mathrm{mg}$ & $93^{\mathrm{a}}$ & $36^{\mathrm{b}}$ & $40^{\mathrm{b}}$ & $36^{\mathrm{b}}$ & $38^{\mathrm{b}}$ & $83^{\mathrm{a}}$ \\
Agar & $50 \mathrm{mg}$ & $91^{\mathrm{a}}$ & $33^{\mathrm{b}}$ & $37^{\mathrm{b}}$ & $33^{\mathrm{b}}$ & $34^{\mathrm{b}}$ & $81^{\mathrm{a}}$ \\
& Control & $95^{\mathrm{a}}$ & $82 \mathrm{a}$ & $91^{\mathrm{a}}$ & $86^{\mathrm{a}}$ & $93^{\mathrm{a}}$ & $85^{\mathrm{a}}$ \\
\hline \multicolumn{2}{c}{ LSD } & 13.622 & 12.454 & 16.410 & 16.07 & 14.544 & 16.880 \\
\multicolumn{2}{c}{ F-value } & $26.14^{*}$ & $15.63^{*}$ & $21.56^{*}$ & $37.71^{*}$ & $22.14^{*}$ & $54.38^{*}$ \\
\hline
\end{tabular}

Means followed by different letters within one column differ significantly at $\mathrm{P}<5 \%$

*Significant at $\mathrm{P}<1 \%$ 


\section{Radical length}

The data revealed that C. album, P. minor and A. fatua showing 52\%, 5\% and 50\% radical length inhibition respectively as compared to control in L. camara leaf powder on filter paper, whereas, no significant effect on radical length of $T$. aestivum, $R$. dentatus and E. helioscopia showing resistance to dry powder. It is also clear from the result that $C$. album, $P$. minor and A. fatua showed 55\%, 53\% and 52\% radical length inhibition respectively as compared to control in powder applied into soil. The results also declared that maximum (98\%) radical length was noted for T. aestivum, $R$. dentatus and E. helioscopia (Table 20). In the present study, it was demonstrated that minimum radical length was noted for $C$. album i.e. $48 \%$ and $45 \%$ on filter paper and soil, respectively. The results of the current study indicated on agar the highest radical length reduction was noted for $P$. minor (36\%), followed by A. fatua (33\%) and C. album (32\%) at $10 \mathrm{mg}$ conc. Similarly, the highest radical length reduction was noted for $C$. album (53\%), followed by P. minor (51\%) A. fatua (50\%) at $50 \mathrm{mg}$ conc. The statistical data concluded that minimum radical length was noted for $P$. minor (64\%) and $C$. album (37\%) at $10 \mathrm{mg}$ and at $50 \mathrm{mg}$ conc., respectively (Fig. 4b).

Table 20. Allelopathic effect of L. camara leaf powder on radical length (cm) of test species

\begin{tabular}{|c|c|c|c|c|c|c|c|}
\hline \multicolumn{2}{|c|}{ Treatments } & \multicolumn{6}{|c|}{ Test species } \\
\hline & & \multirow{2}{*}{ T. aestivum } & \multirow{2}{*}{ A. fatua } & \multirow{2}{*}{$R$. dentatus } & \multirow{2}{*}{ P. minor } & \multirow{2}{*}{ E. helioscopia } & \multirow{2}{*}{ C. album } \\
\hline Media & & & & & & & \\
\hline \multirow{2}{*}{$\begin{array}{l}\text { Filter } \\
\text { paper }\end{array}$} & $10 \mathrm{mg}$ & $9.1^{\mathrm{a}}$ & $4.58^{\mathrm{b}}$ & $7.91^{\mathrm{a}}$ & $4.01^{\mathrm{b}}$ & $9.1^{\mathrm{a}}$ & $4.41^{\mathrm{b}}$ \\
\hline & Control & $9.4^{\mathrm{a}}$ & $9.11^{\mathrm{a}}$ & $7.98^{\mathrm{a}}$ & $8.19^{\mathrm{a}}$ & $9.2^{\mathrm{a}}$ & $9.21^{\mathrm{a}}$ \\
\hline \multirow{2}{*}{ Soil } & $50 \mathrm{mg}$ & $9.3^{\mathrm{a}}$ & $4.36^{\mathrm{b}}$ & $7.94^{\mathrm{a}}$ & $3.86^{\mathrm{b}}$ & $9.3^{\mathrm{a}}$ & $4.26^{\mathrm{b}}$ \\
\hline & Control & $9.6^{\mathrm{a}}$ & $9.13^{\mathrm{a}}$ & $8.00^{\mathrm{a}}$ & $8.21^{\mathrm{a}}$ & $9.4^{\mathrm{a}}$ & $9.41^{\mathrm{a}}$ \\
\hline \multirow{3}{*}{ Agar } & $10 \mathrm{mg}$ & $9.7^{\mathrm{a}}$ & $6.12^{\mathrm{b}}$ & $8.20^{\mathrm{a}}$ & $5.25^{\mathrm{b}}$ & $9.4^{\mathrm{a}}$ & $6.57^{\mathrm{b}}$ \\
\hline & $50 \mathrm{mg}$ & $9.5^{\mathrm{a}}$ & $4.61^{c}$ & $8.19^{a}$ & $4.07^{c}$ & $9.2^{\mathrm{a}}$ & $4.51^{\mathrm{c}}$ \\
\hline & Control & $9.8^{\mathrm{a}}$ & $9.15^{\mathrm{a}}$ & $8.21^{\mathrm{a}}$ & $8.23^{\mathrm{a}}$ & $9.6^{\mathrm{a}}$ & $9.62^{\mathrm{a}}$ \\
\hline \multicolumn{2}{|c|}{ LSD } & 3.2779 & 0.8692 & 0.8236 & 2.5388 & 1.503 & 0.9823 \\
\hline \multicolumn{2}{|c|}{ F-value } & $23.22 *$ & $498.76^{*}$ & $266.70^{*}$ & $47.00 *$ & $57.71 *$ & $66.95 *$ \\
\hline
\end{tabular}

Means followed by different letters within one column differ significantly at $\mathrm{P}<5 \%$

*Significant at $\mathrm{P}<1 \%$

\section{Plumule length}

The data revealed that E. helioscopia, C. album, $R$. dentatus and A. fatua showing $54 \%, 53 \%, 51 \%$ and $50 \%$ plumule length inhibition respectively as compared to control in L. camara leaf powder on filter paper, whereas, no significant effect on plumule length of $T$. aestivum and $P$. minor showing resistance to dry powder. It is also clear from the result that E. helioscopia, C. album, A. fatua and R. dentatus showed 64\%, $61 \%, 59 \%$ and $56 \%$ plumule length inhibition respectively as compared to control in powder applied into soil. The results also declared that maximum (98\%) plumule length was noted for T. aestivum and P. minor. In the present study, it was demonstrated that minimum plumule length was noted for E. helioscopia i.e. $49 \%$ and $36 \%$ on filter paper and soil, respectively (Table 21). The experimental results of the current study indicated on agar the highest plumule length reduction was noted for $R$. dentatus (43\%), followed 
by E. helioscopia (42\%), C. album (42\%) and A. fatua (37\%) at $10 \mathrm{mg}$ conc. Similarly, the highest plumule length reduction was noted for C. album (59\%), followed by $E$. helioscopia (57\%), A. fatua (56\%) and R. dentatus (55\%) at $50 \mathrm{mg}$ conc. The statistical data concluded that minimum plumule length was noted for $R$. dentatus $(67 \%)$ and $C$. album (41\%) at $10 \mathrm{mg}$ and at $50 \mathrm{mg}$ conc., respectively (Fig. $4 c$ ).

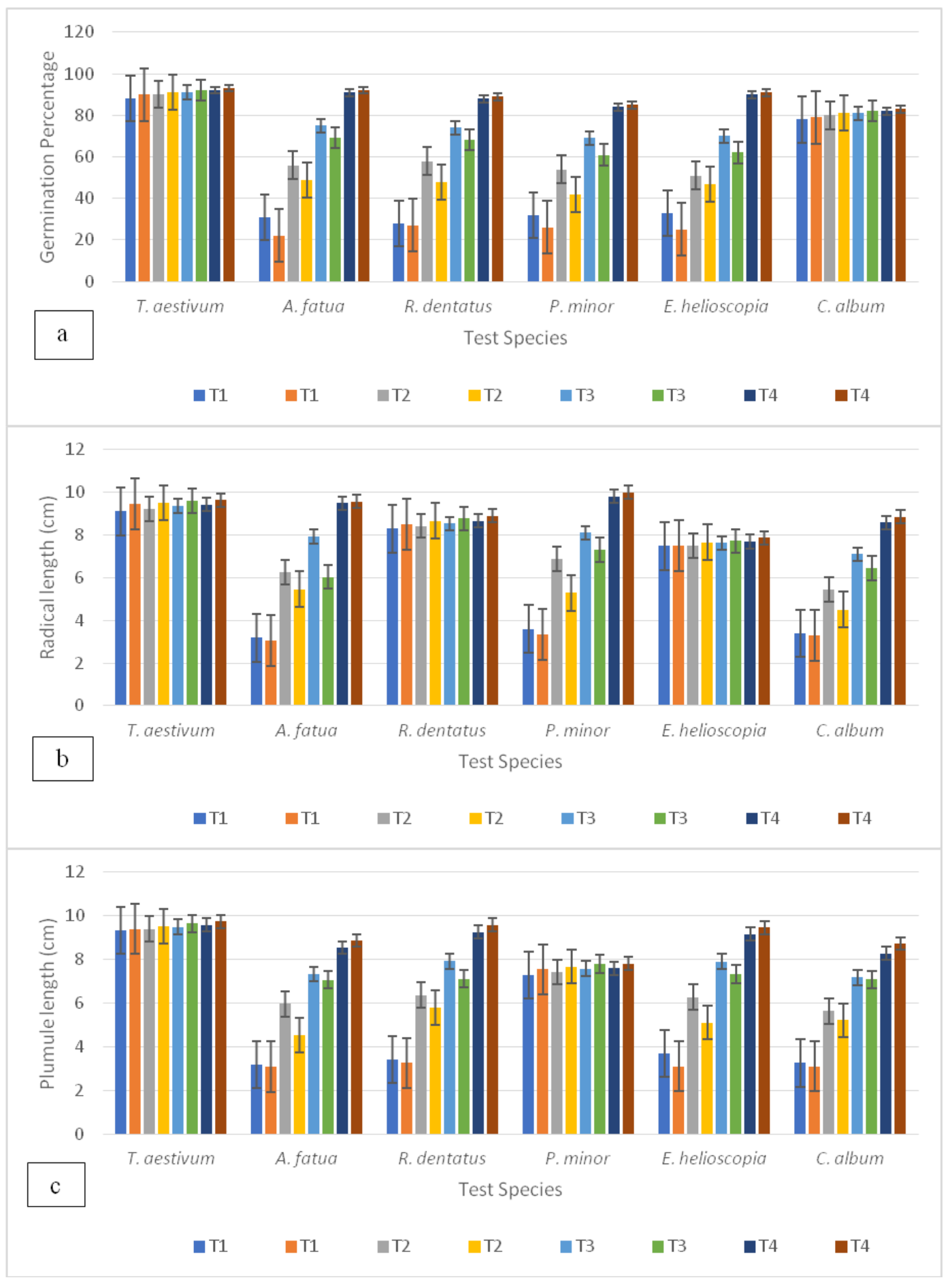

Figure 4. Allelopathic potential of L. camara methanolic extract against test species on filter paper (FP) and soil (S) on: (a) germination percentage (b) radical length (c) plumule length 
Table 21. Allelopathic effect of L. camara leaf powder on plumule length (cm) of test species

\begin{tabular}{|c|c|c|c|c|c|c|c|}
\hline \multicolumn{2}{|c|}{ Treatments } & \multicolumn{6}{|c|}{ Test species } \\
\hline Media & & T. aestivum & A. fatua & R. dentatus & $P$. minor & E. helioscopia & C. album \\
\hline \multirow{2}{*}{$\begin{array}{l}\text { Filter } \\
\text { paper }\end{array}$} & $10 \mathrm{mg}$ & $9.1^{\mathrm{a}}$ & $3.98^{\mathrm{b}}$ & $4.61^{\mathrm{b}}$ & $10.1^{\mathrm{a}}$ & $3.29^{\mathrm{b}}$ & $4.59^{\mathrm{b}}$ \\
\hline & Control & $9.4^{\mathrm{a}}$ & $8.01^{\mathrm{a}}$ & $9.48^{\mathrm{a}}$ & $10.2^{\mathrm{a}}$ & $7.09^{\mathrm{a}}$ & $9.71^{\mathrm{a}}$ \\
\hline \multirow{2}{*}{ Soil } & $50 \mathrm{mg}$ & $9.3^{\mathrm{a}}$ & $3.26^{\mathrm{b}}$ & $3.94^{\mathrm{b}}$ & $10.3^{\mathrm{a}}$ & $3.26^{\mathrm{b}}$ & $3.86^{\mathrm{b}}$ \\
\hline & Control & $9.6^{\mathrm{a}}$ & $8.03^{\mathrm{a}}$ & $9.00^{\mathrm{a}}$ & $10.4^{\mathrm{a}}$ & $9.01^{\mathrm{a}}$ & $10.01^{\mathrm{a}}$ \\
\hline \multirow{3}{*}{ Agar } & $10 \mathrm{mg}$ & $9.7^{\mathrm{a}}$ & $5.02^{b}$ & $5.20^{\mathrm{b}}$ & $10.4^{\mathrm{a}}$ & $4.15^{\mathrm{b}}$ & $5.87^{\mathrm{b}}$ \\
\hline & $50 \mathrm{mg}$ & $9.5^{\mathrm{a}}$ & $3.51^{\mathrm{c}}$ & $4.19^{c}$ & $10.2^{\mathrm{a}}$ & $3.06^{\mathrm{c}}$ & $4.21^{\mathrm{c}}$ \\
\hline & Control & $9.8^{\mathrm{a}}$ & $8.05^{\mathrm{a}}$ & $9.21^{\mathrm{a}}$ & $10.6^{\mathrm{a}}$ & $7.13^{\mathrm{a}}$ & $10.2^{\mathrm{a}}$ \\
\hline \multicolumn{2}{|c|}{ LSD } & 3.4490 & 0.9763 & 1.9841 & 1.3929 & 0.9953 & 0.9834 \\
\hline \multicolumn{2}{|c|}{ F-value } & $21.59 *$ & $128.81^{*}$ & $33.17^{*}$ & $65.06^{*}$ & $51.17^{*}$ & $124.10^{*}$ \\
\hline
\end{tabular}

Means followed by different letters within one column differ significantly at $P<5 \%$

*Significant at $P<1 \%$

\section{Discussion}

Natural herbicides obtained from allelopathic plants can help in reducing usage of synthetic herbicides for weed controlling. Resultantly these may environment friendly better agricultural products as well as alleviate human health concerns (Khan et al., 2014). Lantana camara is reported to possess phenolic, alkaloids and aromatic compounds which inhibit the growth and seed germination of adjacent plant by competing soil nutrients and fluctuating micro environment by producing dense thickets (Dobhal et al., 2010). Keeping this in view, the present study was performed for evaluating L. camara as allelopathic potential against some major weeds (Phalaris minor, Avena fatua, Chenopodium album, Euphorbia helioscopia and Rumex dentatus) of wheat crop.

The aqueous extract of L. camara significantly inhibited seed germination of A. fatua (42\%) and E. helioscopia (36\%) on filter paper and soil, respectively. Besides, there was lowest radical length $(\mathrm{cm})$ in $A$. fatua being the most susceptible weed, while lowermost plumule length was noted in E. helioscopia. These results are in-line with past studies. The aqueous extract of $L$. camara significantly suppressed the growth and seed germination of different plant species including agricultural crops (Sharma et al., 2005; Ahmed et al., 2007). Our results were also in accordance with those of Hussain et al. (2011) who reported that leaf extract L. camara to be the most toxic for test species. Iramus et al. (2011) had observed that the retardation in root length of Vigna radiata was due to inhibitory effect of $L$. camara leaf extract and suppressive effect was concentration dependent. Jabeen and Ahmed (2009) stated that germination and subsequent growth of Cucurbita pepo was significantly reduced by leaf aqueous extract of L. camara. The germination inhibition was due to allelopathic action of some inhibitory chemical substances excreted by plants. Tadele (2014) described that the potential of leaf aqueous extract of L. camara on root and shoot elongation was species specific and concentration dependent. Enyew and Raja (2015) observed that L. camara leaf aqueous extract caused significant retardation in root elongation of Zea mays that may be attributed to the secondary metabolites produced by the extract. The germination and subsequent growth of Eichhornia crassipes was significantly checked 
by aqueous extracts from mature and young leaves of $L$. camara, caused tissues decay and damages in E. crassipes (Saxena, 2000).

The analysis of the data of hexane extract indicated that there was significant reduction in germination of E. helioscopia (35\%), followed by $P$. minor (33\%) and $A$. fatua (32\%) on filter paper, while lowest radical length was observed in A. fatua and the lowermost plumule length in $R$. dentatus. Tadele (2014) stated that leaf hexane extract of L. camara significantly decreased germination of Eragrostis teff. Padhy et al. (2000) described that growth suppression in seed germination and vigor may be due to the presence secondary metabolites in $L$. camara extract. The allelopathic potential of leaf extracts of $L$. camara different concentrations were retarder to all growth parameters of Vigna radiata seeds. The growth of Setaria italica, Lactuca sativa and Pennisetum americanum was significantly checked by all parts of L. camara extracts (Hussain et al., 2011).

The methanolic extract of $L$. camara significantly inhibited seed germination of $R$. dentatus (68\%), A. fatua (66\%), E. helioscopia (63\%) and P. minor (62\%). Besides, the same extract significantly reduced radical length of $A$. fatua and plumule length of $R$. dentatus and E. helioscopia. Mishra (2015) presented the similar results and stated that L. camara has allelopathic effect on Lepidium sativum germination and growth. Ahmed et al. (2007) described the inhibitory potential of L. camara in different crops. Mishra (2012) stated that L. camara extract checked seed germination of Parthenium hysterophorus demonstrating the presence of inhibitory phytochemicals. Oudhia (2000) noticed significant reduction in seed germination of Melilotus alba by extract $L$. camara.

The results of sandwich method indicated significant reduction in germination percentage of A. fatua and E. helioscopia on filter paper and soil by applying L. camara leaf powder. There was no inhibitory effect on $T$. aestivum, $R$. dentatus and $E$. helioscopia. There was minimal radical length in $C$. album and plumule length in $E$. helioscopia that showed the most sensitive weeds against the L. camara leaf powder. Some of the studies reported inhibitory effect of $L$. camara on the germination and growth of various crops. The leaf powder of L. camara inhibited germination, radical and plumule length of radish and lettuce (Qiaoying et al., 2009), P. hysterophorus (Mishra and Singh, 2012), Albizia procera, Acacia auriculiformis, Paraserianthes falcataria, Amaranthus tricolor, Abelmoschus esculentus, Vigna sinensis, Oryza sativa and Cucurbita pepo (Mishra, 2012). Das et al. (2012) also noticed inhibition linked with the production of allelochemicals from the $L$. camara leaves that inhibit growth of adjacent plants by outcompeting for soil nutrients and altering micro environment by forming dense thickets.

\section{Conclusion}

This study was aimed at evaluating allelopathic potential of L. camara against some major weeds of wheat crop. The selected solvent extracts (i.e. aqueous, methanolic and ethanolic) and dried leaf materials significantly inhibited seed germination, radical and plumule length of the selected weeds without harming the wheat plants. Among all the tested extract, methanolic ones showed its supremacy in terms of growth and germination inhibition of the studied weeds. It can be concluded that L. camara methanolic extract can be explored in future studies in purifying and identifying herbicidal compounds to be used in future herbicidal products development. 


\section{REFERENCES}

[1] Ahmed, R., Uddin, M. B., Khan, M. A., Mukul, S. A., Hossain, M. K. (2007): Allelopathic effects of Lantana camara on germination and growth behavior of some agricultural crops in Bangladesh. - Journal Forestry Research 18: 301-304.

[2] Anwar, T., Khalid, S., Arafat, Y., Sadia, S., Riaz, S. (2013): Allelopathic suppression of Avena fatua and Rumex dentatus in associated crops. - Pakistan Journal of Weed Science Research 19: 31-43.

[3] Anwar, T., Khalid, S., Saeed, M., Mazhar, R., Qureshi, H., Rashid, M. (2016): Allelopathic interference of leaf powder and aqueous extracts of hostile weed: Parthenium hysterophorus (Asteraceae). - Science International 4: 86-93.

[4] Anwar, T., Khalid, S., Mazhar, R., Qureshi, H., Rashid, M. (2017a): Herbicidal potential of selected species to overcome weed infestation in Triticum aestivum, Zea mays and Helianthus annuus. - Pakistan Journal of Weed Science Research 23: 49-63.

[5] Anwar, T., Khalid, S., Panni, M. K., Qureshi, H., Rashid, M. (2017b): Allelopathic effect of Euphorbia helioscopia on Avena fatua, Rumex dentatus, Helianthus annuus, Zea mays and Triticum aestivum. - Pakistan Journal of Weed Science Research 23: 165-177.

[6] Arafat, Y., Khalid, S., Lin, W., Fang, C., Sadia, S., Ali, N., Azeem, S. J. (2015): Allelopathic evaluation of selected plants extract against broad and narrow leaves weeds and their associated crops. - Academic Journal of Agricultural Research 3: 226-234.

[7] Das, C. R., Mondal, N. K., Aditya, P., Datta, J. K., Banerjee, A., Das, K. (2012): Allelopathic potentialities of leachates of leaf litter of some selected tree species on gram seeds under laboratory conditions. - Asian Journal of Experimental Biological Science 3: 59-65.

[8] Dobhal, P. K., Kohli, R. K., Batish, D. R. (2010): Evaluation of impact of Lantana camara $\mathrm{L}$. invasion on four major woody shrubs along Nayar river of Pauri Garhwal in Himalaya. - International Journal of Biodiversity and Conservation 2: 166-172.

[9] Enyew, A., Raja, N. (2015): Allelopathic effect of Lantana camara L. leaf powder on germination and growth behavior of maize (Zea mays Linn.) and wheat (Triticum turgidum Linn.) cultivars. - Asian Journal of Agricultural Research 7: 4-10.

[10] Fujii, Y., Parvez, S. S., Parvez, M. M., Ohmae, Y., Iida, O. (2003): Screening of 239 medicinal plant species for allelopathic activity using sandwich method. - Weed Biology Management 3: 233-241.

[11] Fujii, Y., Shibuya, T., Nakatani, K., Itani, T., Hiradate, S., Parvez, M. M. (2004): Assessment method for allelopathic effect from leaf litter leachates. - Weed Biology Management 4: 19-23.

[12] Hussain, M. I., Gonzalez, L., Reigosa, M. J. (2011): Allelopathic potential of Acacia melanoxylon on the germination and root growth of native species. - Weed Biology Management 11: 18-28.

[13] Iramus, S., Moinuddin, A., Syed, T. A. (2011): Allelopathic effect of scarlet pimpernel (Anagallis arvensis) on seed germination and radical elongation of mung bean and pearl millet. - Pakistan Journal of Botany 43: 351-355.

[14] Jabeen, N., Ahmed, M. (2009): Possible allelopathic effect of three different weeds on germination and growth of maize (Zea mays) cultivars. - Pakistan Journal of Botany 41: $1677-1683$.

[15] Khan, I., Ali, Z., Khan, M. I., Hussain, Z., Khan, I. A., Waqas, M., Khan, R. Khan, S. (2014): Allelopathic effects of some weeds on chickpea crop. - Pak. J. Weed Sci. Res. 20: 207-211.

[16] Khan, M. A., Hussain, I., Ahmad, E. (2008): Allelopathic effect of Eucalyptus (Eucalyptus camaldulensis L.) on germination and seedling growth of (Triticum aestivum L.). - Pakistan Journal of Weed Science Research 14: 9-18. 
[17] Maharjan, S., Shrestha, B. B., Jha, P. K. (2007): Allelopathic effects of aqueous extract of Parthenium hysterophorus L. on seed germination and seedling growth of some cultivated and wild herbaceous species. - Science World 5: 35-39.

[18] Michelangelo, M. T., Vidal, R. A., Junior, A. A. B., Bittencourt, H. V. H., Filho, S. S. (2016): Allelopathy: driving mechanisms governing its activity in agriculture. - Journal of Plant Interaction 1: 53-60.

[19] Mishra, A. (2012): Allelopathic interaction of Lantana camara leaf of extract on growth of Parthenium hysterophorus in seedling stage. - International Journal of Plant Science 7: 259-262.

[20] Mishra, A. (2015): Review; allelopathic properties of Lantana camara. - Int Res J Basic Clin Stud 3: 13-28.

[21] Mishra, A. Singh R (2012): Allelopathic effect of Lantana camara extract of different parts on seed germination of Parthenium hysterophorus L. - International Journal of Plant Science 5: 74-75.

[22] Nekonam, M. S., Kraimmojeni, H., Sharifnabi, B., Razmjoo, J., Amini, H., Bahrami, F. (2014): Assessment of some medicinal plants for their allelopathic potential against redroot pigweed (Amaranthus retroflexus). - Journal of Plant Protection and Research 54: 90-95.

[23] Oudhia, P. (2000): Allelopathic effects of some obnoxious weeds on germination of Melilotus alba. - Legume Research 22: 133-134.

[24] Padhy, B., Patnaik, P. K., Tripathy, A. K. (2000): Allelopathic potential of Eucalyptus leaf litter leachate on germination and seedling growth of finger millet. - Allelopathy Journal 7: 69-78.

[25] Qiaoying, Z., Shaolin, P., Yunchun, Z. (2009): Allelopathic potential of reproductive organs of exotic weed Lantana camara. - Allelopathy Journal 23: 213-220.

[26] Qureshi, R., Bhatti, G. R. (2001): Determination of weed communities in wheat (Triticum aestivum Linn.) crop of District Sukkur. - Pakistan Journal of Botany 33(1): 109-115.

[27] Qureshi, R., Waheed, A., Arshad, M. (2009): Weed communities of wheat crop in district Toba Tek Singh, Pakistan. - Pakistan Journal of Botany 41: 239-245.

[28] Raana, G., Khalid, S., Qureshi, R., Sadia, S., Bajwa, A. A. (2012): Screening and evaluation of Euphorbia pulcherrima for weed management. - Pakistan Journal of Weed Science Research 18: 529-539.

[29] Sahu, A., Devkota, A. (2013): Allelopathic effects of aqueous extract of leaves of Mikania micrantha H. B. K. on seed germination and seedling growth of Oryza sativa L. and Raphanus sativus L. - Science World Journal 11: 90-93.

[30] Saxena, M. K. (2000): Aqueous leachate of Lantana camara kills water hyacinth. Journal of Chemical Ecology 26: 2435-2447.

[31] Sharma, G. P., Raghubanshi, A. S., Sing, J. S. (2005): Lantana invasion: an overview. Weed Biology Management 5: 157-167.

[32] Tadele, D. (2014): Allelopathic Effects of Lantana (Lantana camara L.) leaf extracts on germination and early growth of three agricultural crops in Ethiopia. - Momona Ethiopian Journal of Science 6: 111-119.

[33] Zhou, Y. H., Yu, J. Q., Huang, L. F., Nogues, S. (2004): The relationship between $\mathrm{CO}_{2}$ assimilation, photosynthetic electron transport and water-water cycle in chill-exposed cucumber leaves under low light and subsequent recovery. - Plant Cell Environment 27: 1503-1514. 